\title{
Exploring the internal structure of soot particles using nanoindentation: A reactive molecular dynamics study
}

\author{
Laura Pascazio $^{\text {a }}$, Jacob W. Martin ${ }^{\text {a,b }}$, Kimberly Bowal ${ }^{\mathrm{a}}$, Jethro Akroyd ${ }^{\mathrm{a}, \mathrm{b}}$, Markus \\ $\mathrm{Kraft}^{\mathrm{a}} \mathrm{b}, \mathrm{c}, *$ \\ ${ }^{a}$ Department of Chemical Engineering and Biotechnology, University of Cambridge, West Site, \\ Philippa Fawcett Drive, Cambridge, CB3 OAS, United Kingdom \\ ${ }^{b}$ Cambridge Centre for Advanced Research and Education in Singapore (CARES), CREATE \\ Tower, 1 Create Way, Singapore, 138602 \\ ${ }^{c}$ School of Chemical and Biomedical Engineering, Nanyang Technological University, 62 \\ Nanyang Drive, Singapore, 637459
}

\begin{abstract}
The mechanical properties and internal structure of soot nanoparticles is investigated using reactive molecular dynamics simulations of nanoindentation of model soot particles. The particles that are provided as inputs to the simulations are generated using reactive molecular dynamics to create 3D networks of crosslinked coronene, circumanthracene and core-shell mixtures of coronene and circumanthracene. The results of the simulated nanoindentation experiments are analysed as a function of the degree of crosslinking (defined as the number of crosslinks per monomer in the particles), the size and the core-shell structure of the particles. In the case of homogeneous particles (i.e. those without a core-shell structure), the simulations show a unique relationship between the degree of crosslinking $(C L)$ and the simulated hardness, Young's modulus and deformation ratio. In the case of particles with a core-shell structure, a unique relationship was only found by considering the core-shell ratio and the degree of crosslinking in both the core and the shell. Our results allow for interpretation of the nanoindentation experiments
\end{abstract}

\footnotetext{
*Corresponding author

Email address: mk306@cam.ac.uk (Markus Kraft)
} 
as suggesting crosslinks are present in mature soot particles and preliminary evidence that crosslinks also are present within the interior of soot particles. Keywords: molecular dynamics, reactive force field, hardness, crosslinking, nanoindentation, soot

\section{Introduction}

Combustion-generated carbonaceous particles, also known as soot, are generally considered to be unwanted byproducts of combustion processes and a significant atmospheric pollutant $[1,2]$. Over the last decade, many studies have been conducted on the chemical-physical mechanisms responsible for the formation of carbon compounds in flames [3], with the aim of improving combustion efficiency and reducing the emission of pollutants from combustion devices. Moreover, since such carbon compounds share many common characteristics with industrially-produced carbons (graphene, fullerene, carbon black, etc.), a deeper knowledge of the chemistry and morphology of these carbonaceous particles could be a starting point to develop new flame synthesis routes for functional carbon-based nanomaterials [3].

Transmission electron microscopy has long been applied as a tool to study soot structure $[4,5,6]$ and more recently detailed observation and quantification of the internal structure of the particles at the nanoscale can be accessed using high resolution transmission electron microscopy (HR-TEM) and fringe analysis [7, $8,9,10,11]$. Young soot particles $(<10 \mathrm{~nm})$ are characterised by a disordered structure, consisting of short carbon layers with some curvature, which has been interpreted as stacks of polycyclic aromatic hydrocarbon (PAH) molecules [12, 13]. Mature soot primary particles exhibit a core-shell structure consisting of a disordered aromatic particle core, potentially with multiple nuclei [12], containing small fringes surrounded by larger stacked fringes $[14,12,9,13]$. An increase in 
the $\mathrm{C} / \mathrm{H}$ ratio and the degree of crystallinity is observed with soot maturity due to a carbonisation process that is responsible for the growth of the graphitic structures and a decrease in the curvature and interlayer spacing $[15,16]$.

Computational molecular dynamics (MD) and global optimisation methods have also been used to gain insight into the internal structure of soot nanoparticles $[17,18,19]$. Homogeneous clusters containing a single type of PAH molecule are often used as simple analogues of soot particles even though soot particles are known to contain a distribution of PAH molecular sizes [20]. Morphologies of heterogeneous PAH clusters have been recently investigated to understand whether the core-shell structure of soot is due to physical partitioning of different sized PAHs [21]. The simulations found that heterogeneous PAH clusters favour a core-shell structure in which the larger molecules are located closer to the cluster centre. However the inverse morphology was observed experimentally - smaller fringes are found in the core of the particle and larger fringes make up the surface layers - for mature soot particles using $\operatorname{HR}$-TEM $[14,12,9,13]$, indicating that intermolecular interactions are not responsible for the core-shell partitioning seen in mature particles and that crosslinks between PAH molecules might play a crucial role in determining mature soot particle structure. More detailed soot models have also been proposed in literature to represent soot structure. The model proposed by Fernandez-Alos et al. has been created using an automated aromatic structure generation approach directly from HR-TEM lattice fringe images [22]. The models generated by this approach can reproduce the orientation, stacking and distributions of the aromatic moieties within soot but they remain "slice" models that currently do not consider crosslinking and carbonisation.

The presence of crosslinks in soot nanostructures is also important for elucidating information about the very initial stages of soot nanoparticle formation. Reactive aromatic species that crosslink have long been thought to contribute to 
soot inception. A variety of chemical pathways in which PAHs react to form crosslinked three-dimensional structures have been proposed [23, 24, 25, 26, 27] and explored in modelling works [28, 29, 30]. Iavarone et al. showed that the presence of covalent bonds among the aromatic sub-units improves clustering and growth at high temperature [28]. Mao et al. found that only the PAH dimers formed from PAH radicals through radical-radical combination processes possess a lifetime long enough for subsequent PAH growth [30]. Experimental evidence for the chemical structures of aliphatically substituted and bridged PAH species in gas-phase combustion environments has been reported by Adamson et al. [31]. Nuclear magnetic resonance studies on soot formed by pyrolysis have also shown that soot growth consists not only of PAH molecule growth but also PAH crosslinking [32]. High-resolution atomic force microscopy and photoionisation mass spectrometry have recently confirmed the presence of resonancestabilised radical (RSR) species [33, 34, 35]. It has been suggested that these RSR species can undergo crosslinking reactions with a variety of hydrocarbons. The product of these crosslinking reactions can be another RSR species, enabling chain reactions to occur [33].

While imaging crosslinks directly in soot particles has been difficult, nanoindentation can provide insight into the structure of the particles. Nanoindentation is an established technique that measures the force as a well-defined tip is pushed into a material, from which the hardness $(H)$ can be determined. The relative hardness of different carbon materials can provide an indicator for the degree of crosslinking. Nanocrystalline graphite is a noncrosslinked structure made of mobile graphene sheets, possessing very low hardness values (0.1-0.4 GPa) [36]. In contrast, soot shows hardness values of $2-8 \mathrm{GPa}[37,38,39,40]$, values closer to a hard crosslinked structure such as charcoal (3-5 GPa) [41]. The wide range found for soot hardness suggests that particles collected at different residence times and 
produced in different combustion environments may be characterised by different structural properties.

There have been preliminary attempts to incorporate crosslinks into models. Highly graphitised carbon black particles have been constructed from defective concentric fullerene molecules arranged in an onion-like structure with the help of reactive MD $[42,43]$. In conjunction with nanoindentation experiments, a soot particle model was constructed in which the nanoparticle consists of a fully amorphous carbon core and a graphitic shell [40]. This simulation provides initial exploration of the impact of a core and shell structure and the hardness of such particle. However, the model soot particle has a $\mathrm{C} / \mathrm{H}$ ratio of 8 with a 1-to- 1 ratio of the core size to the shell thickness, representative of a mature soot particle. Soot particles are known to contain a significant fraction of hydrogen (unlike materials such as carbon black) and are not completely carbonised, with structural parameters such as shell thickness and particle size that change during their formation and growth. Therefore a new model able to describe soot structure at all of its stages of formation is needed. In a recent paper it was reported that the hardness of crosslinked aromatics increases with the degree of crosslinking in the structure and a range of degrees of crosslinking for ethylene and diesel soot particles was estimated [44]. These hardness values were derived from uniaxial tensile test simulations of periodic boxes containing crosslinked PAHs through the use of an empirical constant of proportionality. The constant was derived by taking into consideration the fact that soot has an intermediate structure between highly oriented pyrolytic graphite and diamond. These results provided ranges for the degree of crosslinking within soot nanoparticles, but did not determine the impact of internal nanostructure or allow for a direct comparison with nanoindentation experiments of soot.

The aim of this paper is to simulate the nanoindentation of model soot par- 
ticles with core-shell structures to provide insights into nanoindentation experiments. The model soot particles that are provided as inputs to the nanoindentation simulations are generated using reactive force field molecular dynamics to create 3D networks of crosslinked PAH arranged in a sphere. To our knowledge, the soot particle model developed here incorporates crosslinks in the soot structure for the first time, where until now the degree of crosslinking has yet to feature prominently in discussions. Structural parameter-mechanical property relationships were derived from nanoindentation simulations of these soot particles. Comparison with nanoindentation experiments performed with in situ transmission electron microscopy provided insights into the degree of crosslinking and the internal nanostructure of soot nanoparticles.

\section{Methodology}

Reactive force field MD simulations were employed to simulate the nanoindentation-induced deformation of model soot particles. All the reactive force field molecular dyanmics simulations were carried out using the LAMMPS software [45]. The interactions within molecules were modelled using an updated version of the adaptive intermolecular reactive empirical bond order (AIREBO) potential [46], known as AIREBO-M [47], which has been widely used to investigate the mechanical and thermal properties of carbon-based nanomaterials $[48,47,49]$. In previous work this method was benchmarked to ensure that it could accurately predict the mechanical properties of PAH molecules [44]. Simulations of tensile tests were used to calculate the Young's modulus for diamond along the $<111>$ and $<100>$ and graphene along the armchair and zig-zag within $4 \%$ of the experimental values $[50,48,51]$. Comparisons between crosslinked biphenyl and naphthalene were slightly overestimated by AIREBO-M (+5.5\% and $+5.0 \%$, respectively) compared with experimental results [52]. The effect of den- 
sity was seen to have a minimal impact on the yield stress and Young's modulus between $1.5-1.8 \mathrm{~g} / \mathrm{cm}^{3}$.

\subsection{Soot particle model construction}

The model soot particles used in this work are PAH clusters with a coreshell structure within which medium-sized aromatic components are crosslinked to varying degrees. Figure 1 illustrates the components of the model particle: a quasi-amorphous central core (coloured in blue in Fig. 1) composed of coronene molecules $\left(\mathrm{C}_{24} \mathrm{H}_{12}\right)$; an ordered outer shell (coloured in grey in Fig. 1) consisting of circumanthracene molecules $\left(\mathrm{C}_{40} \mathrm{H}_{16}\right)$.

[Figure 1 about here.]

These starting molecules were selected to match the size of molecules seen experimentally: HR-TEM and fringe analysis results show that the most abundant PAHs in young and mature soot have approximately 7 aromatic rings (coronene) and the fragment size increases with the radial distance from the centre [13]. The larger circumanthracene molecules have therefore been chosen for the particle shell, and coronene molecules for the core. Note that previously, the size of the constituent molecules was shown to have only a weak effect on mechanical properties whereas a primary dependence was found with the degree of crosslinking [44]. We therefore do not expect different starting monomers or a heterogeneous collection of molecules to modify the results significantly.

Spherical particles of crosslinked molecules with diameters from 7-11 nm were prepared for nanoindentation simulations as follows. PACKMOL software [53] was employed to create starting configurations for the crosslinking process. Monomers were randomly located in the quasi-amorphous core and disposed in layers in the "graphitic" shell. The number of molecules was chosen to give a density of $1.5 \mathrm{~g} / \mathrm{cm}^{3}$, which is a typical value for soot particles [54]. 
The crosslinks between PAH molecules in the particle were created through the same crosslinking procedure that has been developed in a previous publication [44], with core and shell crosslinking reactions run separately. This procedure did not consider a particular crosslinking mechanism as this would require prohibitively long simulations. Instead, reactive sites were generated through the removal of some hydrogen atoms from the rim of the molecule in a manner that allowed single bonds between the aromatics to form. A reactive force field simulation in NVT ensemble was run up to $100 \mathrm{ps}$ and halted at different times to provide varying degrees of crosslinking. A time step of 0.25 fs was adopted for the integration of the atomic equations of motion and the Berendsen thermostat was used to maintain a constant temperature of $1000 \mathrm{~K}$ with a time constant of $0.1 \mathrm{ps}$. These simulation parameters ensured a stable simulation as significant heat had to be removed due to bond formation.

The degree of crosslinking $(C L)$ is defined as:

$$
C L=\frac{2 \cdot \text { number of crosslinks in the system }}{\text { number of starting monomers }} .
$$

$C L$ represents the average number of crosslinks possessed by each molecule in the system. For example, when the $C L$ density is 1 , each molecule has on average a single crosslink and mainly dimers are formed. A $C L$ density of 2 is required for a polymer where on average each molecule has two crosslinks and a $C L$ value higher than 2 produces a 3D network of crosslinked PAHs. The degree of crosslinking increases as a function of the simulation time and is reported in Fig. S1 of the Appendix for all the systems investigated. Hydrogen atoms were then added back to the sites that did not form crosslinks.

At the start of the simulation, monomers are not crosslinked and they interact only physically. PAH monomers are not able to strongly bind together at high temperature with only physical interactions [19]. Due to the high simulation temperature $(1000 \mathrm{~K})$, a position potential was implemented during the reactive force 
field MD simulation to reach the desired degree of crosslinking without the loss of molecules from the particle. This added potential acted on molecules located outside of the particle boundary to restrain them within a spherical volume (core) or spherical slice (shell). The simulations were stopped at the desired degree of crosslinking. PACKMOL software [53] was again used to combine the outer shell with the quasi-amorphous core to produce a single model. It is important to outline that this procedure allows the construction of particles with a desired degree of crosslinking in the core and shell. However, there are no crosslinks between the core and shell components. In order to consider possible crosslinks between the core and the shell, the reactive crosslinking simulations of both cluster components must be run simultaneously, producing a core and a shell with CL values that are nearly the same and cannot be selected and controlled (see Fig. S1 of the Appendix). This is why core and shell crosslinking reactions were run separately in this work.

More than two hundred different particles were constructed to understand the effect of different structural parameters on the simulated hardness. The parameter ranges for the particles considered in this study are summarised in Table 1.

[Table 1 about here.]

\subsection{Nanoindentation simulations}

Reactive force field MD was employed to simulate the nanoindentation-induced deformation of model soot particles in order to investigate the effect of the structural parameters of the particles on their mechanical properties. The particles were first relaxed at $300 \mathrm{~K}$ for $100 \mathrm{ps}$ in the NVT ensemble by using a chain of three Nosé-Hoover thermostats. No position restraint was used at this stage and the particles maintained nearly spherical geometries. The initial velocities of the atoms were assigned in accordance with the Maxwell-Boltzmann distribution. 
Once equilibrated, the nanoparticles were subject to indentation. The setup for the nanoindentation simulations is shown in Fig. 2.

[Figure 2 about here.]

The time step was set as $0.5 \mathrm{fs}$. A virtual wall was placed at the bottom of the particle. The indentation simulations were performed with a planar indenter initially placed $5 \AA$ above the particle. The choice of a planar indenter instead of a spherical one (used in experimental studies) comes from the small size of nanoparticles compared to the typical size of indenter tips. Then, the planar approximation can be used. The simulation was made up of three stages: a loading stage in which the indenter moved downwards at a constant speed of $25 \mathrm{~m} / \mathrm{s}$ (a study of the appropriateness of the indentation speed can be found in Appendix A.3) up to a maximum indentation depth of 0.6 times the initial particle diameter, which is the maximum indentation depth used in soot nanoindentation experiments [40, 39]; a constant load stage in which the indenter was at the maximum indentation depth for $50 \mathrm{ps}$; and an unloading stage in which the indenter moved upwards up to its starting position at the same speed used during the loading stage. The displacement function is reported in Fig. $3 b$.

\subsection{Estimation of mechanical properties}

The mechanical properties of a given material can be derived from the loading/unloading curve [55]. The hardness $(H)$ of the particle can be calculated as follows:

$$
H=\frac{P_{\max }}{A_{\mathrm{c}}}
$$

where $P_{\max }$ is the maximum force acting on the indenter at the end of the loading stage and $A_{\mathrm{c}}$ is the contact area calculated from the atomic positions at the maximum load [55]. The contact area was calculated as the spherical approximation 
of the convex hull of the atoms in contact with the surface of the indenter. This spherical approximation mirrors the experimental procedure [40], which is unable to record the real contact area and instead considers a circle derived from the 2D image recorded during nanoindentation. Additional details about the contact area calculations are reported in Appendix A.2. The nanoindentation curve features thermal fluctuation; for this reason 50 ps of simulation time were added at the maximum load, shown as $\left(t_{2}-t_{1}\right)$ in Fig. 3b. The mean values of $P$ and $A_{\mathrm{c}} \mathrm{ob}-$ tained over this time were used in the calculation $\left(P_{\max }=\bar{P}\left(h_{\max }\right), A_{\mathrm{c}}=\overline{A_{\mathrm{c}}}\left(h_{\max }\right)\right)$. The elastic modulus $(E)$ of the particle can be obtained from the relationship:

$$
\frac{1}{E_{\mathrm{r}}}=\frac{\left(1-v^{2}\right)}{E}+\frac{\left(1-v_{\mathrm{i}}^{2}\right)}{E_{\mathrm{i}}}
$$

where $v$ and $v_{\mathrm{i}}$ are the Poisson's ratios of the particle and the indenter, respectively. $E_{\mathrm{i}}$ is the elastic modulus of the indenter and $E_{\mathrm{r}}$ is the reduced modulus, which takes into account the induced elastic deformations in both the indenter and the particle [55]. For our work, where the indenter was modelled as a fully rigid body, the value of $E_{\mathrm{i}}$ is infinite; thus, the equation simplifies to:

$$
E=E_{\mathrm{r}}\left(1-v^{2}\right)
$$

The Poisson's ratio of the particle was calculated as described in Appendix A.4. The value of $E_{\mathrm{r}}$ is given as:

$$
E_{\mathrm{r}}=\frac{\sqrt{\pi}}{2} \cdot \frac{S_{\max }}{\sqrt{A}}
$$

where $S_{\max }$ is the slope of the unloading curve at the maximum load and $A$ is the contact area at the maximum load [55]. Some of these quantities are shown in Fig. 3a.

[Figure 3 about here.] 


\section{Results}

\subsection{Particles without shell}

The effects of crosslinking and particle diameter on particle mechanical properties are first examined for model soot particles without a surrounding shell (i.e. consisting of a core only). Figure 4 shows the load-displacement $(P-h)$ curves of nanoindentation for these model particles without shells. The particles have a $7 \mathrm{~nm}$ diameter and varying degrees of crosslinking. The particles exhibit elastoplastic behaviour as in previous nanoindentation experiments $[39,37,38]$. It can be observed that the maximum load and the slope of the curves increases with the degree of crosslinking in the particle. The maximum load increases from $0.003 \mu \mathrm{N}$ (noncrosslinked PAH) to $0.33 \mu \mathrm{N}(C L=3.5)$. This is expected since the higher the degree of crosslinking, the stiffer the particle.

[Figure 4 about here.]

From these type of curve it is possible to compute the hardness and Young's modulus using Eq. 2 and 4. These quantities are reported in Fig. 5 for particles of different sizes and with varying degrees of crosslinking. The obtained values all arrange around the same line, indicating that they are not affected by the particle size. The hardness and the Young's modulus increase with the degree of crosslinking, which is consistent with what was obtained from uniaxial tensile test simulations [44]. From a microscopic perspective, this behaviour can be explained as follows: the crosslinking between monomers strengthens the material so that when $C L<2$ (values corresponding to PAH oligomers) the material shows a hardness value lower than $1 \mathrm{GPa}$. It increases almost linearly when $C L>2$ (value corresponding to a $3 \mathrm{D}$ crosslinked structure of $\mathrm{PAH}$ ), up to a value of around $8 \mathrm{GPa}$ when $C L \approx 3.5$. Similar considerations can be made for the Young's modulus $(E)$. The Young's modulus increases with increasing $C L$, which means that the elastic 
recovery is larger at a higher $C L$. The linear dependence of the hardness and the Young's modulus for $C L>2.5$ indicates that every crosslink present in the structure contributes to its mechanical properties, resulting in a direct proportionality relationship.

[Figure 5 about here.]

The Young's modulus is an indicator of a particle's elastic response. However, it cannot be used as an indicator of a particle's plastic behaviour. The hysteresis in the loading/unloading curve indicates that plastic deformation is occurring in the particle. An indicator of the plasticity of the particle is the deformation ratio defined as the ratio between the final and initial height of the particle $\left(z_{\mathrm{f}} / z_{\mathrm{i}}\right)$. Figure 6 shows particles with varying degrees of crosslinking before the loading stage (Fig. 6b), at the maximum load (Fig. 6c) and after the unloading stage (Fig. 6d). When $C L=0$ the final height (height after the unloading stage) of the particle is very similar to the height of the particle at the maximum load, indicating a fully plastic behavior. The ratio between the final and initial heights is around 0.4 times the particle diameter, corresponding exactly to the difference between the initial height and the maximum indentation depth (0.6 times the particle diameter) as reported in Fig. $6 \mathrm{a}$. When $C L \approx 3.5, z_{\mathrm{f}} / z_{\mathrm{i}} \approx 0.9$, indicating that the final height of the particle is almost equal to its initial height. $C L$ values in between these two extremes show an intermediate behaviour, with elasticity increasing with $C L$.

[Figure 6 about here.]

The behaviour of a particle under compression can be compared with the collision of a particle with a surface. AFM experiments on soot particles collected by thermophoretic sampling on a mica disc in a premixed ethylene flame showed that particles with a diameter less than $5 \mathrm{~nm}$ are very flat, while the thickness and 
sphericity increased with the size of the particle, indicating a more rigid structure [56]. These smaller plastic deformations might result from crosslinking reactions involved in particle growth and therefore in an increased $C L$.

In summary, for a particle without a shell, the mechanical properties are a function of the degree of crosslinking in the structure with hardness and elasticity increasing with an increase in $C L$. These properties do not depend on the particle diameter.

\subsection{Core-shell particles}

In this section, we investigate the effect of adding an ordered shell around the PAH crosslinked core. Figure 7 compares the load-displacement curves of particles with a diameter of $9 \mathrm{~nm}$, shell thickness of $1 \mathrm{~nm}, C L_{\text {core }}=2.5$ and varying $C L_{\text {shell }}$ with the response of a shell-free particle with the same size and same $C L_{\text {core }}$ (black line in Fig. 7).

[Figure 7 about here.]

This allows us to explore the effect of different values of $C L_{\text {shell }}$. When $C L_{\text {shell }}<C L_{\text {core }}$, the load shows a delay (defined as the difference in displacement for a specified load) compared to the reference particle with no shell. The core-shell particle provides the same load after a delay that is almost equal to twice the shell thickness when $C L_{\text {shell }}=0$. This delay decreases with $C L_{\text {shell }}$ until $C L_{\text {shell }}=C L_{\text {core }}$ where the two load curves almost overlap. This is expected because when $C L_{\text {shell }} \leq C L_{\text {core }}$ the total degree of crosslinking is lower than or equal to the total degree of crosslinking in the reference particle (shell-free particle with the same size and same $\left.C L_{\text {core }}\right)$. When $C L_{\text {shell }}>C L_{\text {core }}$, core-shell particle load curve provides the same load at a lower indentation depth.

In Fig. 8 the hardness of core-shell particles is compared across different sizes and normalized shell thickness as a function of the total degree of crosslinking. 
[Figure 8 about here.]

The normalized shell thickness is define as the shell thickness-particle radius ratio $\left(d_{\text {shell }} / R_{\mathrm{p}}\right)$. Particles without shell are composed of coronene molecules disposed in a disordered way and they have a value of the normalized shell thickness of zero. Oppositely, a particle composed entirely of circumanthracene molecules disposed in layers has a normalized shell thickness value of 1. Core-shell particle have a normalized shell thickness that goes from 0 to 1 decreasing the core-shell ratio. The total degree of crosslinking is calculated as follows:

$$
C L_{\mathrm{tot}}=\frac{n_{\text {molecules,core }} \cdot C L_{\mathrm{core}}+n_{\text {molecules,shell }} \cdot C L_{\text {shell }}}{n_{\text {molecules,core }}+n_{\text {molecules,shell }}} .
$$

As opposed to the behaviour shown for particles without shells, the hardness values are not simply a function of the total $C L$ in the particle (black line in Fig. 8) and show a large degree of scatter. In particular, at a given value of normalized shell thickness (shown in different colours in Fig. 8), different combinations of degrees of crosslinking in the shell and in the core can give the same hardness value. This suggests that the hardness value is influenced by the distribution of crosslinks in the core and the shell.

In Fig. 9, hardness values for particles with a fixed value for the degree of crosslinking in the core $\left(C L_{\text {core }}=2.5\right)$ are reported as a function of the normalized shell thickness $\left(d_{\text {shell }} / R_{\mathrm{p}}\right)$ and at different $C L_{\text {shell }}$ values. The first thing to note is that when the degree of crosslinking is homogeneously distributed in the particle $\left(C L_{\text {shell }}=C L_{\text {core }}\right)$ the hardness value is not affected by the monomer size in the monomer size range investigated or monomer arrangement since similar hardness values are found within the entire normalized shell thickness range (horizontal line in Fig. 9). This means that the results shown in Fig. 5 are always valid when the particle has a homogeneous degree of crosslinking. Therefore we believe crosslinking effects dominate compared to the influence of local structure 
on particle mechanical properties. This can be explained as follows. When the degree of crosslinking is low, the molecules are highly mobile. So when compressed the molecules rearrange and move easily within the structure, regardless of whether they were originally structured in a disordered way or in stacks. Thus the resulting structures present similar mechanical properties upon indentation. This is also seen in Ref. [39] where different monomer arrangements (case \#2 - parallel stacked graphitic layers normal to the compression direction and case \#4 -stacked graphitic layers whose direction is perpendicular to the compression direction) lead to the same hardness value. When the degree of crosslinking is higher, the crosslinks are formed in all directions within both the core and shell - within the quasi-amorphous core, as well as amongst monomers in the same layer and between layers in the shell. So in both cases we have a 3D network of crosslinks and this leads to similar mechanical properties.

The same results are obtained for a particle with only a core and a core-shell particle with the same homogeneous CL value, suggesting that the inclusion of crosslinks between the core and the shell in our model (which is currently not taken into account) would not significantly alter the results reported.

Conversely, when the degree of crosslinking is not homogeneous $\left(C L_{\text {shell }} \neq C L_{\text {core }}\right)$, the particle hardness value is comparatively higher or lower. Another observation from Fig. 9 is that when the shell becomes big enough $\left(d_{\text {shell }} / R_{\mathrm{p}}>0.7\right)$ the hardness curve reaches a plateau and the shell completely shields the effect of the core, giving the same results as the homogeneous case where $C L$ is equal to $C L_{\text {shell }}$. Therefore, the mechanical properties are mainly dependent on the particle surface properties. In fact, a very thin shell can produce considerable variations in the mechanical properties and a very thick shell can completely shield the contribution of the structural properties of the core on the mechanical properties of the particle.

[Figure 9 about here.] 
Finally, all the data obtained for more than 200 particles with different structural parameters (particle diameter, core/shell ratio, $C L_{\text {core }}$ and $C L_{\text {shell }}$ ) have been fitted to contoured 3D surface plots and reported in Fig. 10. These plots show the dependence of hardness, Young's modulus and deformation ratio $\left(z_{\mathrm{f}} / z_{\mathrm{i}}\right)$ on the degree of crosslinking in the core and the shell for particles with three different values of normalized shell thickness $(0.22,0.37$ and 0.5$)$. As it is possible to note, when the normalized shell thickness is low (left-hand column) the properties are strongly dependent on both the $C L_{\text {core }}$ and $C L_{\text {shell }}$, and as the shell thickness increases, the shell contribution becomes more important. In fact, when the normalized shell thickness is 0.5 it is already clear that the mechanical properties are mainly dictated by the degree of crosslinking in the shell. These contour plots can be used to estimate the degree of crosslinking in the particle from experimental studies of the mechanical and structural properties of soot particles, as discussed in the next section.

[Figure 10 about here.]

\section{Discussion}

We now summarise the simulation results for the purpose of interpreting experimental nanoindentation studies as follows.

For a particle in which the shell is not yet formed:

1. Particles with low $C L$ values have a very low hardness. The hardness increases as a function of the $C L$ in the structure. This is linear with $C L$ when $C L$ is higher than 2.

2. The mechanical properties (hardness, Young's modulus) do not depend on the particle diameter. 
3. The mechanical properties do not depend on the size of the starting monomer or monomer arrangement in the monomer size range investigated, indicating that the shell-free particle results can be used also for particles where the degree of crosslinking is homogeneously distributed throughout the particle, irrespective of the distribution of monomers within the particle.

For a core-shell particle:

1. The mechanical properties depend on $C L_{\text {core }}, C L_{\text {shell }}$ and the core/shell ratio.

2. When the shell becomes large enough $\left(d_{\text {shell }} / R_{\mathrm{p}}>0.7\right)$, it shields the effect of the core.

These results provide guidelines to aid in relating the mechanical properties and the internal nanostructure of soot particles. In the following, we related the results of free-shell particles composed of coronene molecules as representative of young soot particle and core-shell particles composed of coronene (core) and circumanthracene (shell) as representative of mature soot. The choice of the coronenecircumanthracene systems for mature soot has been motivated in Section 2. Young soot, however, show larger fringes on the outside and smaller fringes in the central part of the particle $[13,21]$. However, the difference in size of PAH in the central and outer part in young soot particle is not as large as in mature soot (few aromatic rings compared to almost double the number of rings respectively), so that this assumption remains reasonable.

With the knowledge of these relationships, it is possible to estimate ranges for the degree of crosslinking in the core and shell of a soot particle, given its shell thickness and mechanical properties. The figure shows results for a diesel soot particle tested by nanoindentation (particle D1B in Ref. [40]), with the relevant experimental details and crosslinking results shown in Fig. 11. The particle has a hardness value of $2.5 \mathrm{GPa}$ and Young's modulus of $21 \mathrm{GPa}$ (see Appendix A.5 for full details of the analysis). HR-TEM images of this particle also 
allow the evaluation of the normalized shell thickness $\left(d_{\text {shell }} / R_{\mathrm{p}} \approx 0.4\right)$ and deformation ratio $\left(z_{\mathrm{f}} / z_{\mathrm{i}}=0.84\right)$. The contour plot with the closest $d_{\text {shell }} / R_{\mathrm{p}}$ ratio $\left(d_{\text {shell }} / R_{\mathrm{p}}=0.37\right)$ has been selected from Fig. 10. From this contour plot, it was possible to extract hardness, Young's modulus and deformation ratio contour lines with values of particle D1B in the $C L_{\text {core }}-C L_{\text {shell }}$ plane. The ranges of $C L_{\text {core }}$ and $C L_{\text {shell }}$ that give those values of mechanical properties across the different simulations of particles with same $d_{\text {shell }} / R_{\mathrm{p}}$ are also reported (shaded areas). The region where the contours intersect shows the range of possible values for the degrees of crosslinking of the core and shell for the soot D1B particle $\left(C L_{\text {core }}=1.5-3\right.$ and $\left.C L_{\text {shell }}=2.6-3.4\right)$.

[Figure 11 about here.]

A simulated TEM image of a particle with degree of crosslinking in the core and shell in the range found for particle D1B has also been reported in Fig. 11 (see Appendix A.5 for further details on the method adopted for the TEM image simulation). Unfortunately, we were only able to determine detailed crosslinking information for one particle. All other experiments reported were not made on primary particles but on small aggregates (such as D1A or D2C in [40]) and/or the load displacement curve were not reported.

Another nanoindentation study provides experimental results that are used to estimate the degree of crosslinking for soot particles at different stages in the flame [37]. In Fig. 12, experimental values of the hardness, particle diameter, shell thickness and core diameter for ethylene soot particles at different heights above the burner (HAB) are shown in red.

[Figure 12 about here.]

Soot particle size increased with the height above the burner up to $\mathrm{HAB}=5.0 \mathrm{~cm}$, followed by a reduction in size due to oxidation at a longer distance from the flame 
base. The particles taken at $\mathrm{HAB}=0.5 \mathrm{~cm}$ were disordered and have no shell. Above $\mathrm{HAB}=0.5 \mathrm{~cm}$, the diameters of the particle cores were reduced and the shell thicknesses increased with increasing $\mathrm{HAB}$ until $5 \mathrm{~cm}$. When $\mathrm{HAB}=7.0 \mathrm{~cm}$ a distinct central (spherical) core is no longer visible. The hardness was $3.5 \mathrm{GPa}$ for the particle at $\mathrm{HAB}=0.5 \mathrm{~cm}$ and it decreased slightly $(H=3.0 \mathrm{GPa}$ at $\mathrm{HAB}=2.0 \mathrm{~cm}$ ) when the shell started to form, only to increase again up to a value of 4.5 at $\mathrm{HAB}=7.0 \mathrm{~cm}$. Using the results for a particle composed of only a core (Fig. 5) and assuming that they are not dependent on the monomer size or arrangement (as suggested from our results in Fig. 9), the degree of crosslinking of the particles collected at $0.5 \mathrm{~cm}$ and $7.0 \mathrm{~cm}$ were estimated from their experimental hardness values and found to be 2.8 and 3.1, respectively. In the case of a core-shell particle, the degree of crosslinking in the core and shell are two free parameters that cannot be both estimated from the particle hardness alone. One parameter must be assumed to estimate the other. Two possible behavioural assumptions can be assigned to particles with a shell. The first is that the degree of crosslinking does not increase when the shell starts to form (case 1 in Fig. 12) and the second is that the degree of crosslinking in the core increases with the heights above the burner (case 2 in Fig. 12). It is unlikely that the degree of crosslinking in the core decreases with the heights above the burner, knowing that the particles at the top of the flame are found to have a higher degree of crosslinking compared to those at the bottom. Starting from these assumptions, it is possible to estimate the degree of crosslinking in the shell at the required normalized shell thickness using a linear fitting between those obtained from the contour plots in Fig. 10 at different normalized shell thickness. The values are marked in green in Fig. 12 if subject to assumptions and in blue if not subject to assumptions. The degrees of crosslinking in the core and in the shell are around 2.8 and 2.6 respectively for the particles collected at $\mathrm{HAB}=2.0 \mathrm{~cm}$. At $\mathrm{HAB}=5.0 \mathrm{~cm}$, the normalized 
shell thickness is higher than 0.7 such that the contribution of the core to the overall hardness is negligible and the degree of crosslinking in the shell can be again obtained from the homogeneous case.

The construction of these model soot particles as systems containing only two types of regions (i.e. the core and the shell) is also an assumption. Mechanical properties are strongly dependent on the distribution of crosslinks within the particle and therefore more regions with varied $C L$ values, reflecting many layers composed of many different degrees of crosslinking that may be present in soot structures, may influence the results. However, although our results are subject to a variety of assumptions, we consider that these assumptions are reasonable for a preliminary exploration of the internal structure inaccessible to HR-TEM. Additional techniques would greatly improve the methodologies presented here, such as solid state NMR or atomic force microscopy studies. One general result is that while the simulated hardness values for crosslinked PAH clusters can range from very near to zero to $11 \mathrm{GPa}$ for $C L=0-4$, from experimental soot hardness the degree of crosslinking of mature soot particles are all found to lie between 2.6 and 3.1, indicating a significant number of crosslinks and the importance of considering crosslinks when developing accurate soot models.

A phenomenological model of soot formation can also be suggested from these results. Soot nuclei (1-3 nm) are thought to be formed by the collision of PAH, either in the form of individual molecules or radicals or clusters of molecules or radicals. The incipient particle contain species that may interact chemically (i.e. PAHs connected by aliphatic bonds) and physically (i.e. formation of stacks of polyaromatic units) and have a degree of crosslinking between 0 and 2. As shown in Section 3.1, these particles are characterised by low hardness values and deform plastically. This is consistent with the liquid-like behaviour that incipient soot particles show at high temperatures. Further increases in particle size are presumed 
to occur by the condensation of PAHs from the gas-phase and the formation of a 3D network of crosslinked PAHs, with the degree of crosslinking increasing as they move up the flame. The development of a core-shell structure comes from two different processes: 1) the decrease in the core diameter suggests that some of the aliphatic components surronding the/in the core graphitise after the formation of the initial shell, with the same effect also being responsible for the fringe length increase observed in HR-TEM experiments; 2) the increase in particle size and the lower degree of crosslinking found in the shell with respect to the core and its increase traversing the flame suggests that PAHs of bigger sizes are still condensing from the gas-phase. At the later stages, oxidation is responsible for the particle size decrease.

Unfortunately only few nanoindentation experiments were performed on soot particles. We hope that our results can motivate more experimental nanoindentation work in order to elucidate a clearer description of what occurs during soot formation, especially with regards to young soot particles and particles with a thin shell around their core. These will shed light on the mechanism involved in the first stages of core and shell formation.

Finally, it is worth noting that the presence of five-membered rings, aliphatic chains, asymmetric groups, curvature, or larger monomers might affect mechanical properties, due to potential variations in microstructure, anisotropy, molecular properties, crosslink scheme, etc. This study focuses primarily on the effect of the degree of crosslinking which is made possible only by controlling other factors that could lead to misleading conclusions, such as different monomer types. Thus the choice of two monomers that are very similar in size and of the same type are appropriate for a first study of this kind. We can conclude that the degree of crosslinking seems to be a major factor influencing the mechanical properties and the presence of crosslinks are a mandatory condition in order to observe mechan- 
ical properties similar to those reported by experiments. A more general model that will include different monomer types will be developed and investigated in a future work.

\section{Conclusions}

The mechanical properties of model soot particles are simulated using reactive force field molecular dynamics to investigate their mechanical properties as a function of the degree of crosslinking (defined as the number of crosslinks per monomer in the particles) and the size and core-shell structure of the particles. The particles that are provided as inputs to the simulations are generated using reactive force field molecular dynamics to create 3D networks of crosslinked coronene, circumanthracene and core-shell mixtures of coronene and circumanthracene.

In the case of homogeneous particles (i.e. those without a core-shell structure), the simulations show a unique relationship between the degree of crosslinking and the simulated hardness, Young's modulus and deformation ratio. The relationship is independent of particle size, monomer size and monomer arrangement. In the case of particles with a core-shell structure, a unique relationship was only found by considering the core-shell ratio and the degree of crosslinking in both the core and the shell. It was observed that the mechanical properties are mainly dependent on the particle surface properties. In fact, even a very thin shell was able to cause a large change in the properties of the particles and a very thick shell was able to completely shield the contribution of the structural properties of the core on the mechanical properties of the particle.

Our results allows, in the context of nanoindentation and HR-TEM experiments, the degree of crosslinking in soot particles to be estimated. Despite the assumptions made in the presented model, the results clearly suggest internal crosslinking within soot particles. Further simulations will provide further in- 
sights into the impact of crosslink types and further experiments could provide insights into early soot nanoparticles degree of crosslinking.

\section{Acknowledgements}

This project is supported by the National Research Foundation (NRF), Prime Minister's Office, Singapore under its Campus for Research Excellence and Technological Enterprise (CREATE) programme. This project has received funding from the European Union's Horizon 2020 Research and Innovation Programme under grant agreement no. 724145. The authors are grateful to EPSRC (grant number: EP/R029369/1) and ARCHER for financial and computational support as a part of their funding to the UK Consortium on Turbulent Reacting Flows (www.ukctrf.com). 


\section{References}

[1] T. C. Bond, S. J. Doherty, D. W. Fahey, P. M. Forster, T. Berntsen, B. J. Deangelo, et al., Bounding the role of black carbon in the climate system: A scientific assessment, Journal of Geophysical Research Atmospheres 118 (11) (2013) 5380-5552. doi:10.1002/jgrd.50171.

[2] P. J. Landrigan, R. Fuller, N. J. R. Acosta, O. Adeyi, R. Arnold, N. N. Basu, et al., The Lancet Commission on pollution and health, The Lancet. doi: $10.1016 / \mathrm{S} 0140-6736(17) 32345-0$.

[3] H. Wang, Formation of nascent soot and other condensed-phase materials in flames, Proceedings of the Combustion Institute 33 (1) (2011) 41-67. doi:10.1016/j.proci.2010.09.009.

[4] R. A. Dobbins, C. M. Megaridis, Morphology of flame-generated soot as determined by thermophoretic sampling, Langmuir 3 (2) (1987) 254-259. doi:10.1021/la00074a019.

[5] R. Vander Wal, A TEM methodology for the study of soot particle structure, Combustion Science and Technology 126 (1-6) (1997) 333-351. doi:10. $1080 / 00102209708935680$.

[6] S. di Stasio, Electron microscopy evidence of aggregation under three different size scales for soot nanoparticles in flame, Carbon 39 (1) (2001) 109_ 118. doi:10.1016/S0008-6223(00)00099-3.

[7] R. H. Shim, H. S.and Hurt, N. Yang, A methodology for analysis of 002 lattice fringe images and its application to combustion-derived carbons, Carbon 38 (1) (2000) 29-45. doi : 10.1016/S0008-6223(99)00096-2. 
[8] K. Yehliu, R. L. Vander Wal, A. L. Boehman, Development of an HR-TEM image analysis method to quantify carbon nanostructure, Combustion and Flame 158 (9) (2011) 1837-1851. doi :10.1016/j . combustflame. 2011. 01.009.

[9] M. L. Botero, D. Chen, S. González-Calera, D. Jefferson, M. Kraft, HRTEM evaluation of soot particles produced by the non-premixed combustion of liquid fuels, Carbon 96 (2016) 459-473. doi : 10.1016/j . carbon. 2015.09 .077 .

[10] C. Wang, T. Huddle, C. H. Huang, W. Zhu, R. L. Vander Wal, E. H. Lester, J. P. Mathews, Improved quantification of curvature in high-resolution transmission electron microscopy lattice fringe micrographs of soots, Carbon 117 (2017) 174-181. doi:10.1016/j. carbon.2017.02.059.

[11] M. L. Botero, N. Eaves, J. A. Dreyer, Y. Sheng, J. Akroyd, W. Yang, M. Kraft, Experimental and numerical study of the evolution of soot primary particles in a diffusion flame, Proceedings of the Combustion Institute 37 (2019) 2047-2055. doi : doi .org/10.1016/j .proci.2018.06 . 185.

[12] T. Ishiguro, Y. Takatori, K. Akihama, Microstructure of diesel soot particles probed by electron microscopy: First observation of inner core and outer shell, Combustion and Flame 108 (1) (1997) 231-234. doi :10.1016/ S0010-2180(96)00206-4.

[13] M. L. Botero, Y. Sheng, J. Akroyd, J. W. Martin, J. A. H. Dreyer, W. Yang, M. Kraft, Internal structure of soot particles in a diffusion flame, Carbon 141 (2019) 635-642. doi:10.1016/j. carbon.2018.09.063.

[14] B. Apicella, P. Pré, M. Alfè, A. Ciajolo, V. Gargiulo, C. Russo, A. Tregrossi, D. Deldique, J. N. Rouzaud, Soot nanostructure evolution in pre- 
mixed flames by high resolution electron transmission microscopy (HRTEM), Proceedings of the Combustion Institute 35 (2) (2015) 1895-1902. doi:10.1016/j.proci.2014.06.121.

[15] A. Oberlin, Carbonization and graphitization, Carbon 22 (6) (1984) 521541. doi:10.1016/0008-6223(84)90086-1.

[16] K. Hayashida, S. Nagaoka, H. Ishitani, Growth and oxidation of graphitic crystallites in soot particles within a laminar diffusion flame, Fuel 128 (6) (2014) 148-154. doi:10.1016/j.fuel.2014.03.008.

[17] L. Pascazio, M. Sirignano, A. D’Anna, Simulating the morphology of clusters of polycyclic aromatic hydrocarbons: The influence of the intermolecular potential, Combustion and Flame 185 (2017) 53-62. doi:10.1016/j. combustflame.2017.07.003.

[18] M. Rapacioli, F. Calvo, F. Spiegelman, C. Joblin, D. J. Wales, Stacked clusters of polycyclic aromatic hydrocarbon molecules, The Journal of Physical Chemistry A 109 (11) (2005) 2487-2497. doi : 10.1021/jp046745z.

[19] T. S. Totton, A. J. Misquitta, M. Kraft, A quantitative study of the clustering of polycyclic aromatic hydrocarbons at high temperatures., Physical Chemistry Chemical Physics 14 (12) (2012) 4081-4094. doi:10.1039/ c2cp23008a.

[20] M. L. Botero, E. M. Adkins, S. González-Calera, H. Miller, M. Kraft, PAH structure analysis of soot in a non-premixed flame using high-resolution transmission electron microscopy and optical band gap analysis, Combustion and Flame 164 (2016) 250-258. doi:10.1016/j.combustflame. 2015.11.022. 
[21] K. Bowal, J. W. Martin, M. Kraft, Partitioning of polycyclic aromatic hydrocarbons in heterogeneous clusters, Carbon 143 (2019) 247-256. doi : $10.1016 / j$. carbon. 2018.11.004.

[22] V. Fernandez-Alos, J. K. Watson, R. vander Wal, J. P. Mathews, Soot and char molecular representations generated directly from HR-TEM lattice fringe images using Fringe3D, Combustion and Flame 158 (9) (2011) 1807-1813. doi:10.1016/j.combustflame.2011.01.003.

[23] A. D'Anna, A. Violi, A. D'Alessio, A. F. Sarofim, A reaction pathway for nanoparticle formation in rich premixed flames, Combustion and Flame 127 (1-2) (2001) 1995-2003. doi:10.1016/S0010-2180(01)00303-0.

[24] A. Violi, A. Kubota, T. Truong, W. Pitz, C. Westbrook, A. Sarofim, A fully integrated kinetic Monte Carlo/molecular dynamics approach for the simulation of soot precursor growth, Proceedings of the Combustion Institute 29 (2) (2002) 2343-2349. doi:10.1016/S1540-7489 (02)80285-1.

[25] A. Violi, A. F. Sarofim, G. A. Voth, Kinetic Monte Carlo-molecular dynamics approach to model soot inception, Combustion Science and Technology 176 (5-6) (2004) 991-1005. doi :10 .1080/00102200490428594.

[26] M. Commodo, G. De Falco, A. Bruno, C. Borriello, P. Minutolo, A. D’ Anna, Physicochemical evolution of nascent soot particles in a laminar premixed flame: from nucleation to early growth, Combustion and Flame 162 (10) (2015) 3854-3863. doi:10.1016/j . combustflame.2015.07.022.

[27] K. O. Johansson, T. Dillstrom, P. Elvati, M. F. Campbell, P. E. Schrader, D. M. Popolan-vaida, N. Richards-Henderson, K. Wilson, A. Violi, H. A. Michelsen, Radical-radical reactions, pyrene nucleation, and incipient soot 
formation in combustion, Proceedings of the Combustion Institute 36 (1) (2017) 799-806. doi:10.1016/j.proci.2016.07.130.

[28] S. Iavarone, L. Pascazio, M. Sirignano, A. De Candia, A. Fierro, L. de Arcangelis, A. D'Anna, Molecular dynamics simulations of incipient carbonaceous nanoparticle formation at flame conditions, Combustion Theory and Modelling 21 (1) (2017) 49-61. doi : 10.1080/13647830 . 2016.1242156.

[29] Q. Mao, A. C. T. van Duin, K. H. Luo, Formation of incipient soot particles from polycyclic aromatic hydrocarbons: A ReaxFF molecular dynamics study, Carbon 121 (2017) 380-388. doi:10.1016/j. carbon. 2017. 06.009 .

[30] Q. Mao, D. Hou, K. H. Luo, X. You, Dimerization of polycyclic aromatic hydrocarbon molecules and radicals under flame conditions, Journal of Physical Chemistry A 122 (2018) 8701-8708. doi : 10.1021/acs . jpca. 8b07102.

[31] B. D. Adamson, S. A. Skeen, M. Ahmed, N. Hansen, Detection of aliphatically bridged multi-core polycyclic aromatic hydrocarbons in sooting flames with atmospheric-sampling high-resolution tandem mass spectrometry, Journal of Physical Chemistry A 122 (48) (2018) 9338-9349. doi:10.1021/ acs. jpca. 8b08947.

[32] M. S. Solum, A. F. Sarofim, R. J. Pugmire, T. H. Fletcher, H. Zhang, ${ }^{13}$ C NMR analysis of soot produced from model compounds and a coal, Energy Fuels 15 (2001) 961-971. doi:10.1021/ef0100294?rand=qp6ntlo5.

[33] K. O. Johansson, M. P. Head-Gordon, P. E. Schrader, K. R. Wilson, H. A. Michelsen, Resonance-stabilized hydrocarbon-radical chain reactions may 
explain soot inception and growth, Science 361 (2018) 997-1000. doi: 10.1126/science.aat3417.

[34] M. Commodo, K. Kaiser, G. De Falco, P. Minutolo, F. Schulz, A. D’ Anna, L. Gross, On the early stages of soot formation: Molecular structure elucidation by high-resolution atomic force microscopy, Combustion and Flame 205 (2019) 154-164. doi : 10.1016/j.combustflame.2019.03.042.

[35] F. Schulz, M. Commodo, K. Kaiser, G. De Falco, P. Minutolo, G. Meyer, A. D’Anna, L. Gross, Insights into incipient soot formation by atomic force microscopy, Proceedings of the Combustion Institute 37 (1) (2019) 885-892. doi:10.1016/j.proci.2018.06.100.

[36] J. R. Patterson, S. A. Catledge, Y. K. Vohra, J. Akella, S. T. Weir, Electrical and mechanical properties of $\mathrm{C}_{70}$ fullerene and graphite under high pressures studied using designer diamond anvils, Physical Review Letters 85 (25) (2000) 5364-5367. doi:10.1103/PhysRevLett.85.5364.

[37] H. Bhowmick, S. K. Biswas, Relationship between physical structure and tribology of single soot particles generated by burning ethylene, Tribology Letters 44 (2) (2011) 139-149. doi:10.1007/s11249-011-9831-5.

[38] H. Bhowmick, S. K. Majumdar, S. K. Biswas, Dry tribology and nanomechanics of gaseous flame soot in comparison with carbon black and diesel soot, Proceedings of the Institution of Mechanical Engineers, Part C: Journal of Mechanical Engineering Science 226 (2) (2011) 394-402. doi: 10.1177/0954406211429410.

[39] I. Z. Jenei, F. Dassenoy, T. Epicier, A. Khajeh, A. Martini, D. Uy, H. Ghaedni, A. Gangopadhyay, Mechanical response of gasoline soot 
nanoparticles under compression: An in situ TEM study, Tribology International 131 (2019) 446-453. doi:10.1016/j.triboint.2018.11.001.

[40] I. Z. Jenei, F. Dassenoy, T. Epicier, A. Khajeh, A. Martini, D. Uy, et al., Mechanical characterization of diesel soot nanoparticles: in situ compression in a transmission electron microscope and simulations, Nanotechnology 29 (2018) 085703. doi:10.1088/1361-6528/aaa2aa.

[41] G. A. Zickler, T. Schöberl, O. Paris, Mechanical properties of pyrolysed wood: a nanoindentation study, Philosophical Magazine 86 (10) (2006) 1373-1386. doi: 10.1080/14786430500431390.

[42] G. Hantal, S. Picaud, P. N. Hoang, V. P. Voloshin, N. N. Medvedev, P. Jedlovszky, Water adsorption isotherms on porous onionlike carbonaceous particles. Simulations with the grand canonical Monte Carlo method, The Journal of Chemical Physics 133 (14) (2010) 144702. doi:10.1063/1. 3496466.

[43] C. G. Fernández, S. Picaud, M. Devel, Calculations of the mass absorption cross sections for carbonaceous nanoparticles modeling soot, Journal of Quantitative Spectroscopy and Radiative Transfer 164 (2015) 69 - 81. doi:10.1016/j.jqsrt.2015.05.011.

[44] L. Pascazio, J. W. Martin, M. L. Botero, M. Sirignano, A. D’Anna, M. Kraft, Mechanical properties of soot particles: the impact of crosslinked polycyclic aromatic hydrocarbons, Combustion Science and Technologydoi: $10.1080 / 00102202.2019 .1668380$.

[45] S. Plimpton, Fast parallel algorithms for short-range molecular dynamics, Journal of Computational Physics 117 (1) (1995) 1-19. doi:10.1006/ jcph.1995.1039. 
[46] S. J. Stuart, A. B. Tutein, J. A. Harrison, A reactive potential for hydrocarbons with intermolecular interactions, The Journal of Chemical Physics 112 (14) (2000) 6472-6486. doi:10.1063/1.481208.

[47] T. C. O'Connor, J. Andzelm, M. O. Robbins, AIREBO-M: A reactive model for hydrocarbons at extreme pressures, The Journal of Chemical Physics 142 (2) (2015) 024903. doi : 10 .1063/1.4905549.

[48] H. Zhao, K. Min, N. R. Aluru, Size and Chirality Dependent Elastic Properties of Graphene Nanoribbons under Uniaxial Tension, Nano Letters 9 (8) (2009) 3012-3015. doi : 10.1021/n1901448z.

[49] K. Rego, V. Meunier, Carbon nanotube knots, AIP Advances 9 (2019) 025030. doi:10.1063/1.5088145.

[50] H. Richter, T. G. Benish, O. A. Mazyar, W. H. Green, J. B. Howard, Formation of polycyclic aromatic hydrocarbons and their radicals in a nearly sooting premixed benzene flame, Proceedings of the Combustion Institute 28 (2) (2000) 2609-2618. doi:10.1016/S0082-0784(00)80679-7.

[51] C. Lee, X. Wei, J. Kysar, J. Hone, Measurement of the elastic properties and intrinsic strength of monolayer graphene, Science 321 (2008) 385-388. doi:10.1126/science.1157996.

[52] R. S. Tranter, S. J. Klippenstein, L. B. Harding, B. R. Giri, X. Yang, J. H. Kiefer, Experimental and theoretical investigation of the self-reaction of phenyl radicals, The Journal of Physical Chemistry A 114 (32) (2010) 82408261. doi:10.1021/jp1031064.

[53] L. Martinez, R. Andrade, E. G. Birgin, J. M. Martínez, PACKMOL: A package for building initial configurations for molecular dynamics simu- 
lations, Journal of Computational Chemistry 30 (13) (2009) 2157-2164. doi:10.1002/jcc.21224.

[54] B. Zhao, K. Uchikawa, H. Wang, A comparative study of nanoparticles in premixed flames by scanning mobility particle sizer, small angle neutron scattering, and transmission electron microscopy, Proceedings of the Combustion Institute 31 (2007) 851-860. doi:10.1016/j.proci.2006.08. 064.

[55] W. C. Oliver, G. M. Pharr, Measurement of hardness and elastic modulus by instrumented indentation: Advances in understanding and refinements to methodology, J. Mater. Res. 19 (1) (2004) 3-20. doi :10.1557/jmr . 2004. 19.1.3.

[56] M. Commodo, G. De Falco, P. Minutolo, A. D'Anna, Structure and size of soot nanoparticles in laminar premixed flames at different equivalence ratios, Fuel 216 (2018) 456-462. doi :10.1016/j.fuel.2017.12.032. 


\section{List of Figures}

1 Structure of a model soot particle consisting of a quasi-amorphous core and an ordered outer shell. Blue and grey colours indicate carbon atoms in the core and shell, respectively. . . . . . . . 36

2 Molecular dynamics model of a particle of crosslinked coronene molecules for nanoindentation with a planar indenter. . . . . . . 37

3 (a) Illustration of the load-displacement curve and the parameters used in the calculations of hardness and Young's modulus. (b) The displacement-time function used during the indentation simulations. . . . . . . . . . . . . . . . 38

4 The load-displacement curves of particles with $7 \mathrm{~nm}$ diameter, without shells and with varying degrees of crosslinking $(C L)$. . . 39

5 (a) Hardness $(H)$ and (b) Young's modulus $(E)$ as a function of the degree of crosslinking in the particle $(C L)$. Different colours correspond to different particle sizes. The black lines and grey areas are provided to show the value ranges across the different simulations. . . . . . . . . . . . . . .

6 a) The deformation ratio $\left(z_{\mathrm{f}} / z_{\mathrm{i}}\right)$ as a function of the degree of crosslinking in the particle $(C L)$. Different colours correspond to different particle sizes. The black line and grey shading are guides to visualise the trend and range of values obtained for the $z_{\mathrm{f}} / z_{\mathrm{i}}$ values. b) Screenshots of particles with varying degrees of crosslinking before the loading stage, c) at the maximum load and d) and after the unloading stage. . . . . . . . . . . . . . . . 41

7 The load-displacement curves of particles with a $9 \mathrm{~nm}$ diameter, $C L_{\text {core }}=2.5, d_{\text {shell }}=1 \mathrm{~nm}$ and with varying degrees of crosslinking in the shell $\left(C L_{\text {shell }}\right)$. The black line refers to a shell-free particle with the same size $(9 \mathrm{~nm})$ and $C L_{\text {core }}=2.5 \ldots \ldots \ldots$

8 Hardness $(H)$ as a function of the total degree of crosslinking in the particle $\left(C L_{\text {tot }}\right)$. Different colours correspond to different normalized shell thickness. The black line refers to particles without shells. . . . . . . . . . . . . . . . . 43

9 Hardness $(H)$ as a function of the normalized shell thickness $\left(d_{\text {shell }} / R_{\mathrm{p}}\right)$ for particles with $C L_{\text {core }}=2.5$. Different colours correspond to particles with different $C L_{\text {shell }}$ values. . . . . . . . . . . . 44

10 Hardness $(H)$ (first row), Young's modulus $(E)$ (second row) and deformation ratio $\left(z_{f} / z_{i}\right)$ (third row) as a function of the degree of crosslinking in the core $\left(C L_{\text {core }}\right)$ and the shell $\left(C L_{\text {shell }}\right)$ at three different normalized shell thickness $\left(d_{\text {shell }} / R_{\mathrm{p}}\right): 0.22$ (first column), 0.37 (second column) and 0.5 (third column). . . . . . . . . . . 45 
11 Hardness $(H)$ (red), Young's modulus $(E)$ (blue) and deformation ratio $\left(z_{\mathrm{f}} / z_{\mathrm{i}}\right)$ (green) contour lines with error bounds (shaded areas) in the $C L_{\text {core }}-C L_{\text {shell }}$ plane for the particle D1B tested with nanoindentation in Ref. [40]. The striped area indicates the range of possible values of $C L_{\text {core }}$ and $C L_{\text {shell }}$ for the particle D1B. A table that summarizes the mechanical and structural properties of particle D1B and a simulated TEM image of a particle with degree of crosslinking in the core and shell in the range found for particle D1B has also been reported. . . . . . . . . . . . . . 46

12 Experimental values (red) taken from Ref. [37] of the average particle radius $\left(R_{\mathrm{p}}\right)$, core radius $\left(R_{\text {core }}\right)$, shell thickness $\left(d_{\text {shell }}\right)$, normalized shell thickness $\left(d_{\text {shell }} / R_{\mathrm{p}}\right)$ and hardness $(H)$ of ethylene soot particles collected at different heights above the burner (HAB) and the estimated (blue) and assumed (green) degrees of crosslinking in the core $\left(C L_{\text {core }}\right)$ and in the shell $\left(C L_{\text {shell }}\right) \ldots \ldots . .47$ 


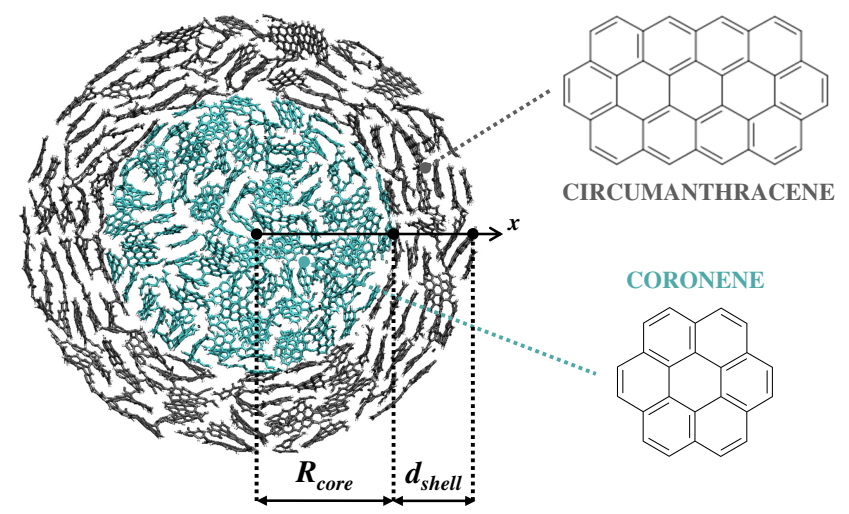

Figure 1: Structure of a model soot particle consisting of a quasi-amorphous core and an ordered outer shell. Blue and grey colours indicate carbon atoms in the core and shell, respectively. 


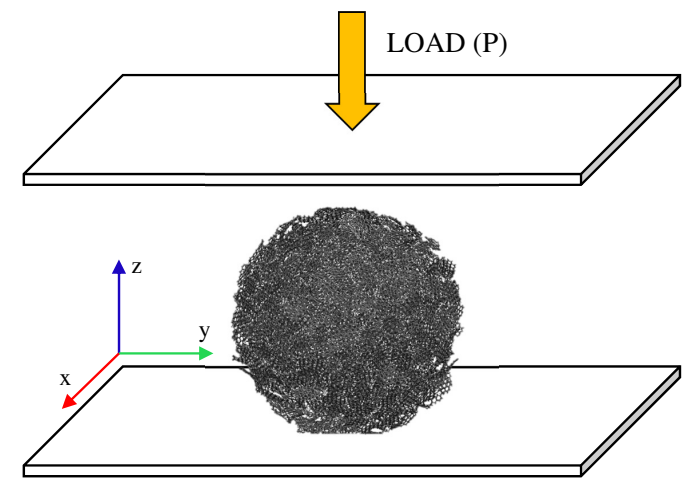

Figure 2: Molecular dynamics model of a particle of crosslinked coronene molecules for nanoindentation with a planar indenter. 


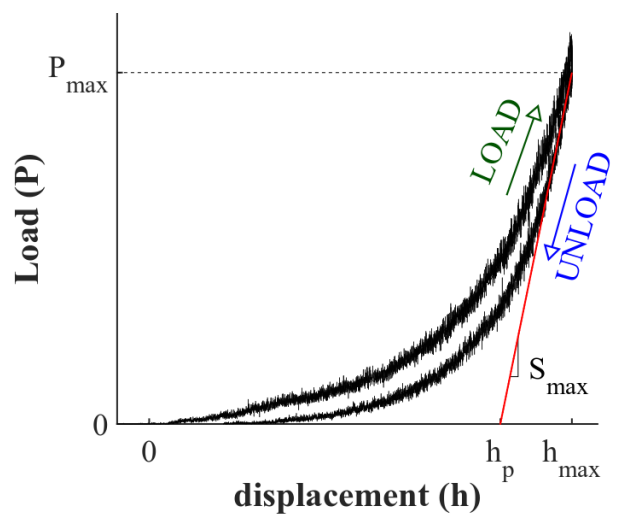

(a)

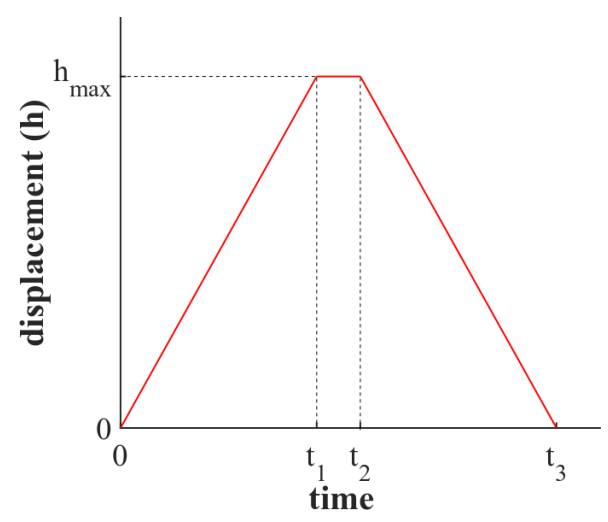

(b)

Figure 3: (a) Illustration of the load-displacement curve and the parameters used in the calculations of hardness and Young's modulus. (b) The displacement-time function used during the indentation simulations. 


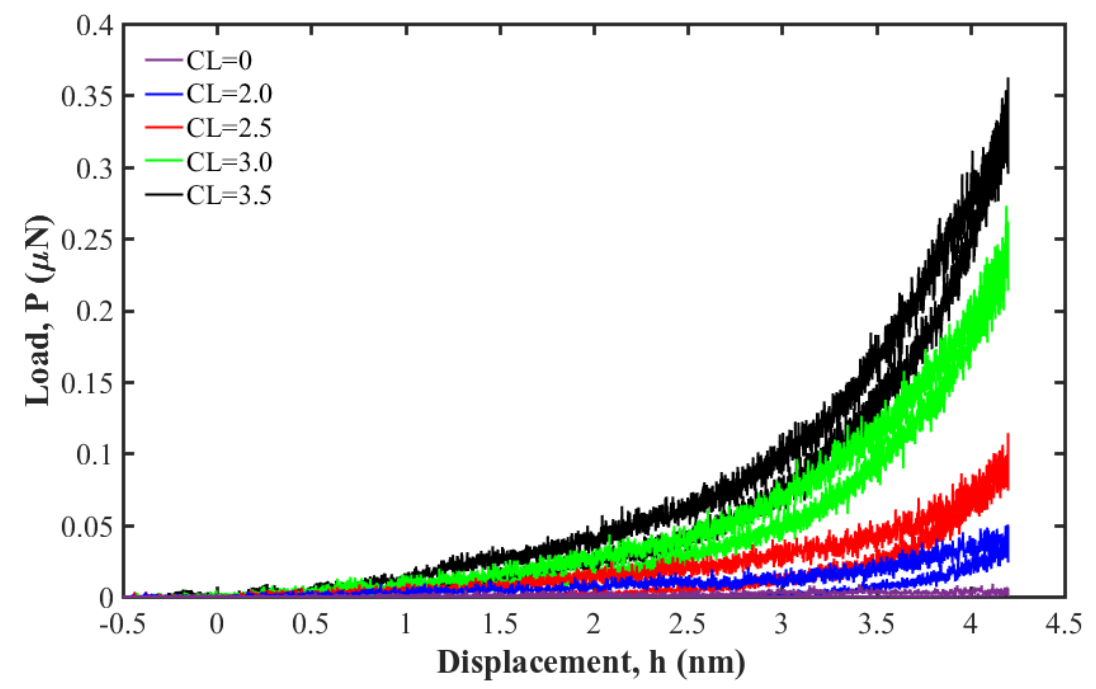

Figure 4: The load-displacement curves of particles with $7 \mathrm{~nm}$ diameter, without shells and with varying degrees of crosslinking $(C L)$. 
(a)

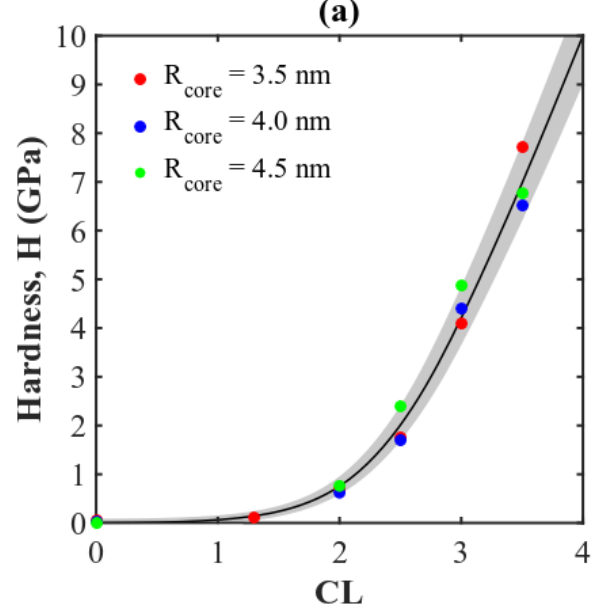

(b)

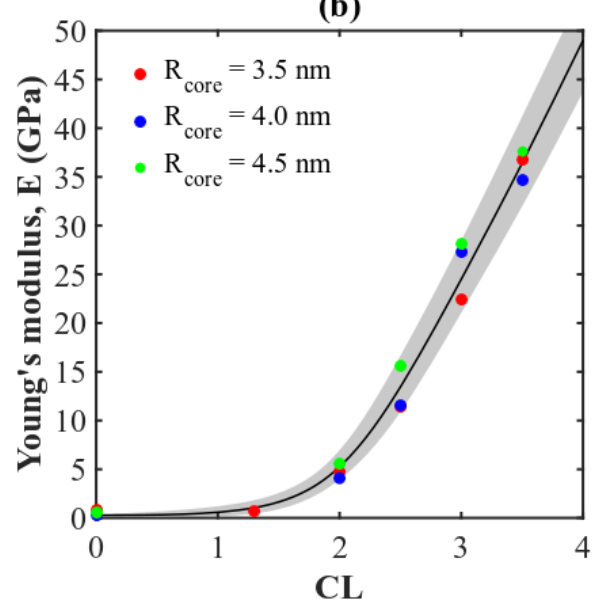

Figure 5: (a) Hardness $(H)$ and (b) Young's modulus $(E)$ as a function of the degree of crosslinking in the particle $(C L)$. Different colours correspond to different particle sizes. The black lines and grey areas are provided to show the value ranges across the different simulations. 
(a)

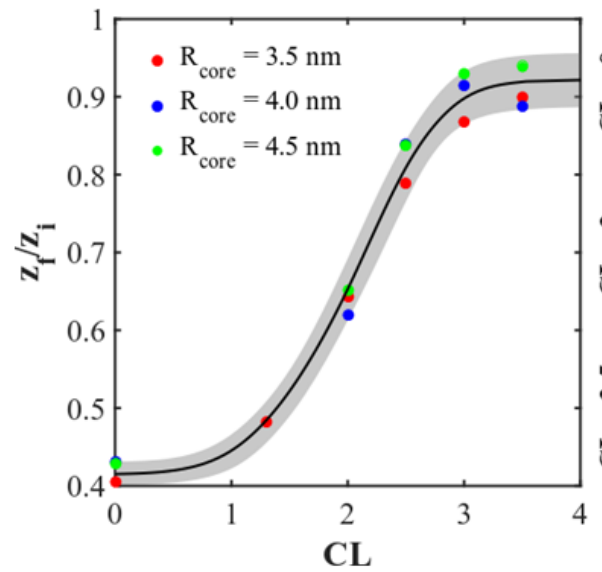

(b)

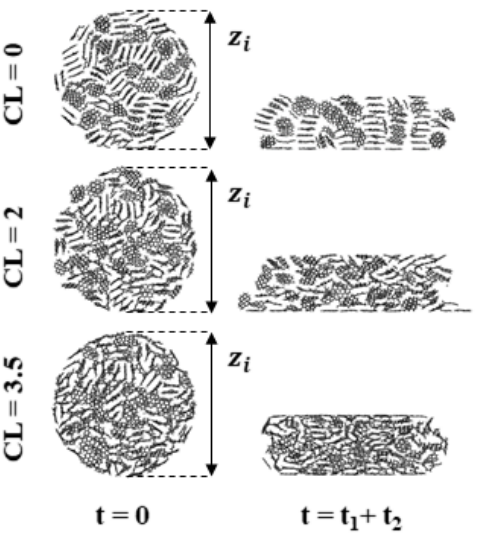

(d)

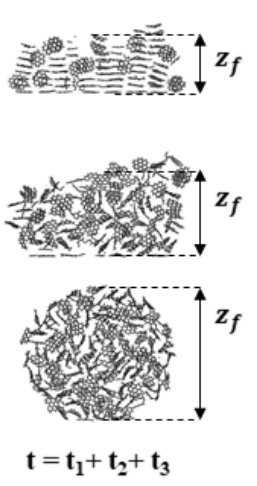

Figure 6: a) The deformation ratio $\left(z_{\mathrm{f}} / z_{\mathrm{i}}\right)$ as a function of the degree of crosslinking in the particle $(C L)$. Different colours correspond to different particle sizes. The black line and grey shading are guides to visualise the trend and range of values obtained for the $z_{\mathrm{f}} / z_{\mathrm{i}}$ values. b) Screenshots of particles with varying degrees of crosslinking before the loading stage, $c$ ) at the maximum load and d) and after the unloading stage. 


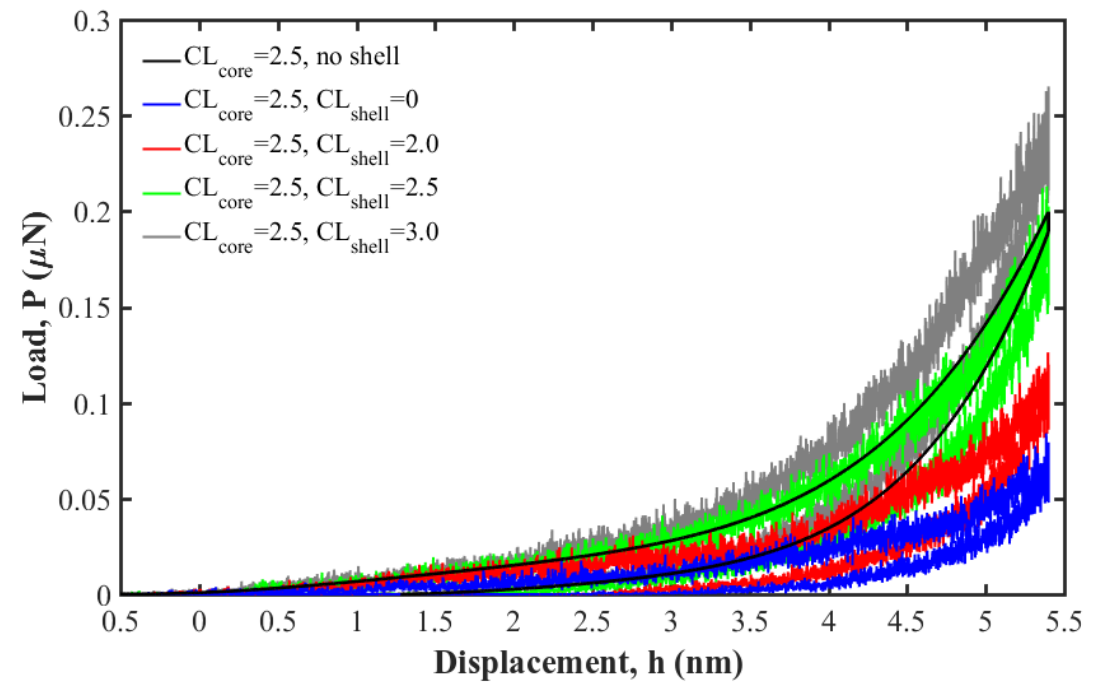

Figure 7: The load-displacement curves of particles with a $9 \mathrm{~nm}$ diameter, $C L_{\text {core }}=2.5$, $d_{\text {shell }}=1 \mathrm{~nm}$ and with varying degrees of crosslinking in the shell $\left(C L_{\text {shell }}\right)$. The black line refers to a shell-free particle with the same size $(9 \mathrm{~nm})$ and $C L_{\text {core }}=2.5$. 


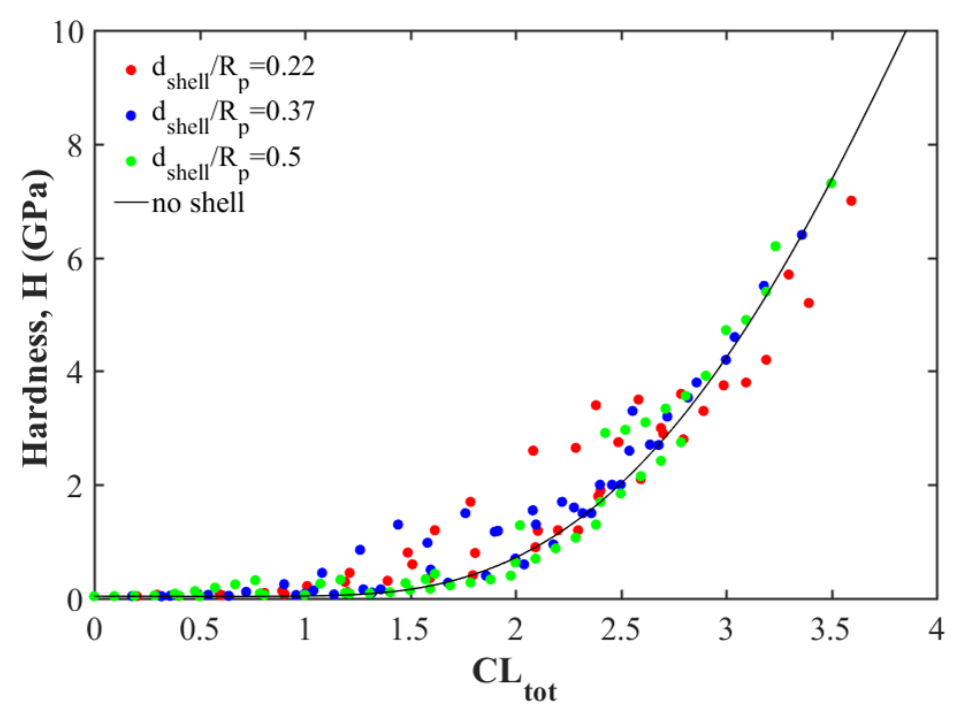

Figure 8: Hardness $(H)$ as a function of the total degree of crosslinking in the particle $\left(C L_{\mathrm{tot}}\right)$. Different colours correspond to different normalized shell thickness. The black line refers to particles without shells. 


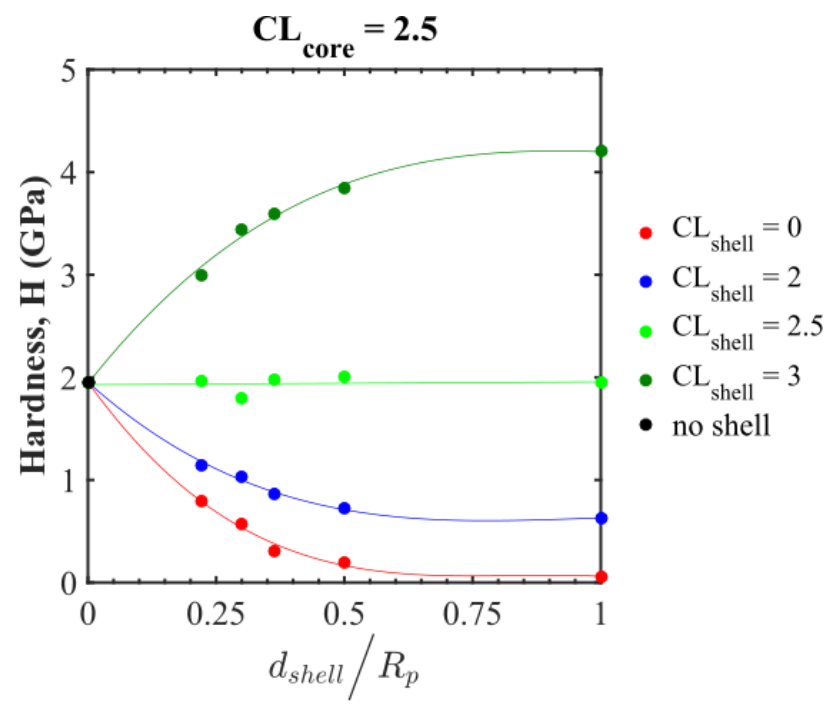

Figure 9: Hardness $(H)$ as a function of the normalized shell thickness $\left(d_{\text {shell }} / R_{\mathrm{p}}\right)$ for particles with $C L_{\text {core }}=2.5$. Different colours correspond to particles with different $C L_{\text {shell }}$ values. 

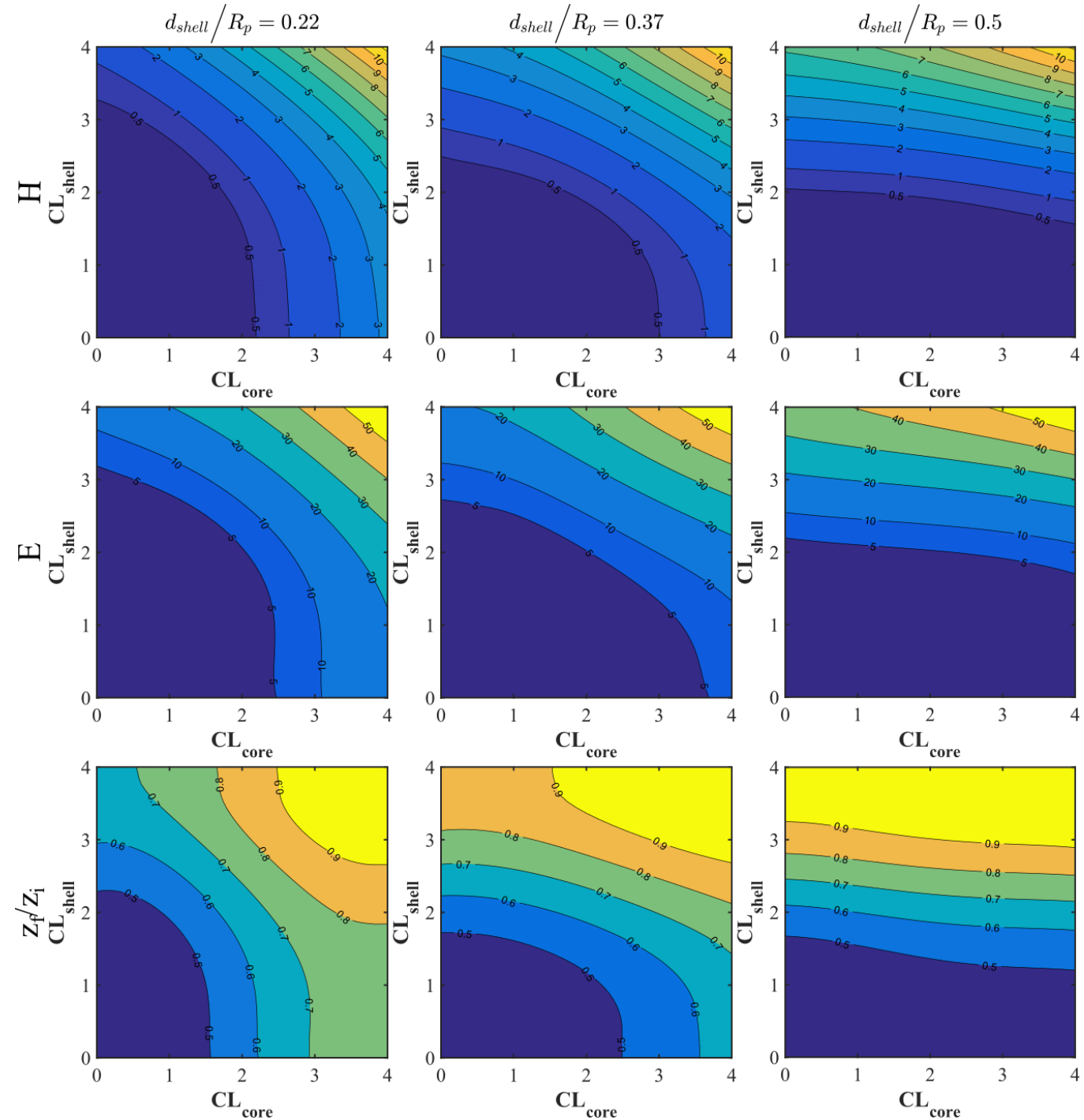

Figure 10: Hardness $(H)$ (first row), Young's modulus $(E)$ (second row) and deformation ratio $\left(z_{f} / z_{i}\right)$ (third row) as a function of the degree of crosslinking in the core $\left(C L_{\text {core }}\right)$ and the shell $\left(C L_{\text {shell }}\right)$ at three different normalized shell thickness $\left(d_{\text {shell }} / R_{\mathrm{p}}\right): 0.22$ (first column), 0.37 (second column) and 0.5 (third column). 


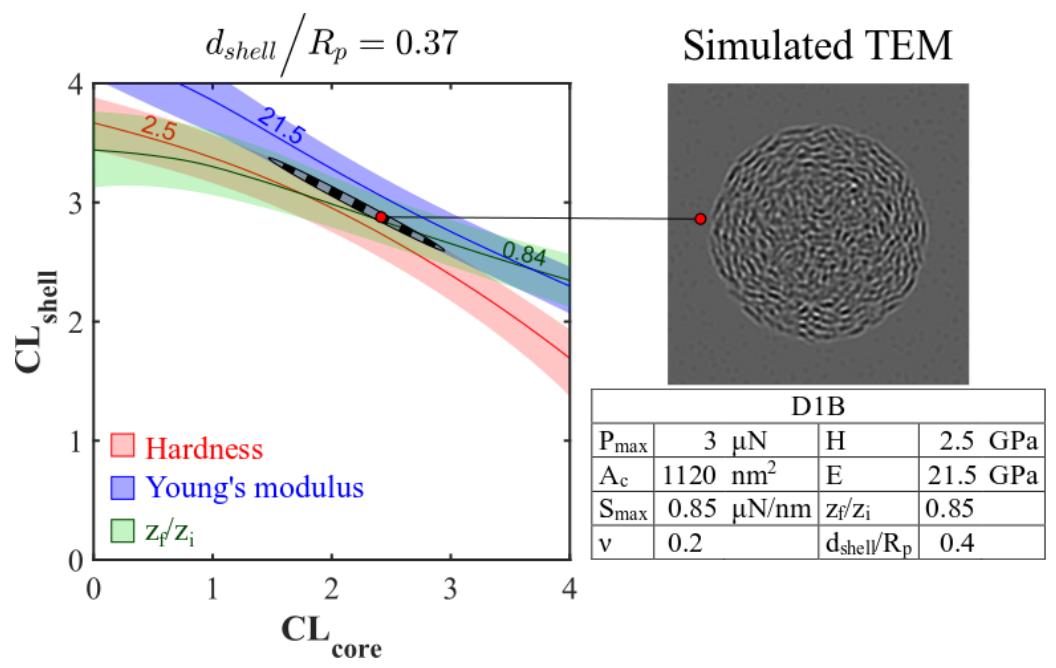

Figure 11: Hardness $(H)\left(\right.$ red), Young's modulus $(E)$ (blue) and deformation ratio $\left(z_{\mathrm{f}} / z_{\mathrm{i}}\right)$ (green) contour lines with error bounds (shaded areas) in the $C L_{\text {core }}-C L_{\text {shell }}$ plane for the particle D1B tested with nanoindentation in Ref. [40]. The striped area indicates the range of possible values of $C L_{\text {core }}$ and $C L_{\text {shell }}$ for the particle D1B. A table that summarizes the mechanical and structural properties of particle D1B and a simulated TEM image of a particle with degree of crosslinking in the core and shell in the range found for particle D1B has also been reported. 


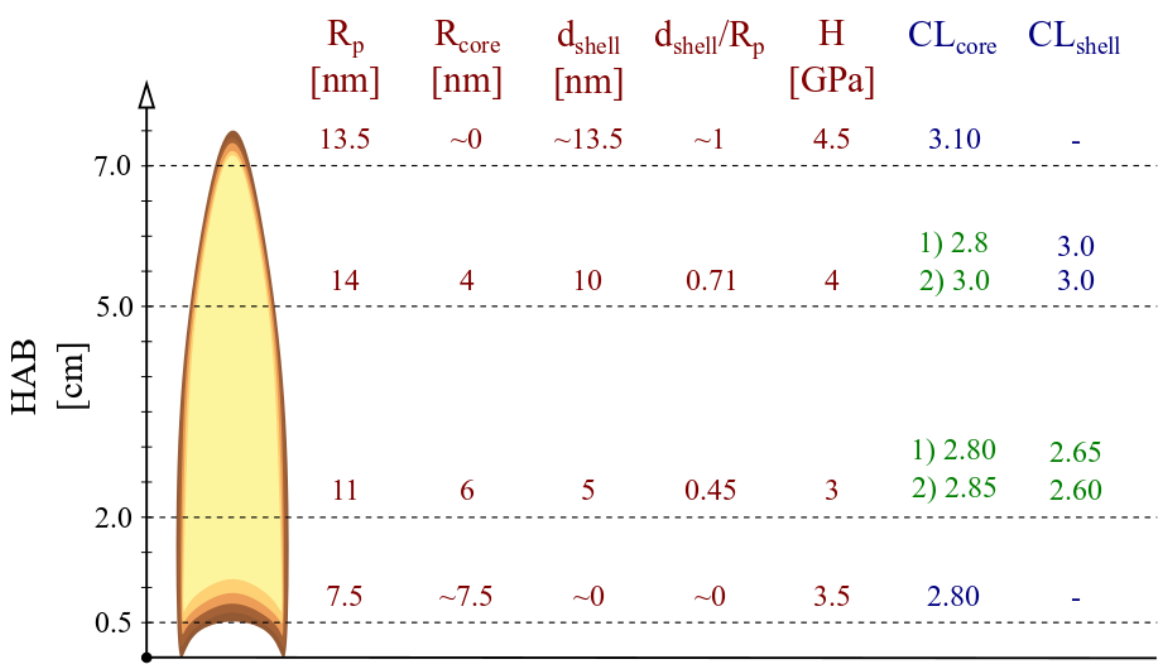

Figure 12: Experimental values (red) taken from Ref. [37] of the average particle radius $\left(R_{\mathrm{p}}\right)$, core radius $\left(R_{\text {core }}\right)$, shell thickness $\left(d_{\text {shell }}\right)$, normalized shell thickness $\left(d_{\text {shell }} / R_{\mathrm{p}}\right)$ and hardness $(H)$ of ethylene soot particles collected at different heights above the burner (HAB) and the estimated (blue) and assumed (green) degrees of crosslinking in the core $\left(C L_{\text {core }}\right)$ and in the shell $\left(C L_{\text {shell }}\right)$. 


\section{List of Tables}

1 Structural parameters of the investigated model soot particles: degree of crosslinking in the core $\left(C L_{\text {core }}\right)$, degree of crosslinking in the shell $\left(C L_{\text {shell }}\right)$, core radius $\left(R_{\text {core }}\right)$, shell thickness $\left(d_{\text {shell }}\right)$ and particle radius $\left(R_{\mathrm{p}}\right) \ldots \ldots \ldots \ldots \ldots$ 
Table 1: Structural parameters of the investigated model soot particles: degree of crosslinking in the core $\left(C L_{\text {core }}\right)$, degree of crosslinking in the shell $\left(C L_{\text {shell }}\right)$, core radius $\left(R_{\text {core }}\right)$, shell thickness $\left(d_{\text {shell }}\right)$ and particle radius $\left(R_{\mathrm{p}}\right)$.

\begin{tabular}{l|c|c|c}
\hline Parameter & Min & Max & Units \\
\hline$C L_{\text {core }}$ & 0.00 & 4.00 & - \\
$C L_{\text {shell }}$ & 0.00 & 3.30 & - \\
$R_{\text {core }}$ & 0.00 & 4.50 & $\mathrm{~nm}$ \\
$d_{\text {shell }}$ & 0.00 & 4.50 & $\mathrm{~nm}$ \\
$R_{\mathrm{p}}$ & 3.50 & 5.50 & $\mathrm{~nm}$
\end{tabular}

